\title{
Un aménagement piscicole réussi, celui du barrage Aube
}

\author{
H. Colin*
}

\section{Introduction}

Le barrage Aube a été réalisé dans le cadre du programme de régularisation de la Seine et de ses principaux affluents en amont de Paris. Il vient d'être mis en service en 1990. Cinq autres ouvrages ont été mis en service auparavant :

- le réservoir de Crescent ;

- le réservoir de Chauneçon ;

- le réservoir de Pannesière-Chaumard;

et en Champagne humide :

- le réservoir Seine (205 millions de $\mathrm{m}^{3}$ ) exploité depuis 1966 ;

- le réservoir Marne (350 millions de $\mathrm{m}^{3}$ ) mis en service en janvier 74 .

Ces deux derniers ouvrages sont très proches du réservoir Aube et ont fait l'objet d'études piscicoles au début des années 80. Réservoirs Seine et Aube sont dans le même département de l'Aube. La Fédération des Associations Agréées de Pêche et de Pisciculture du département de l'Aube avait donc quelques idées quant à des aménagements piscicoles d'un milieu qui serait semblable au réservoir Seine.

Les grands réservoirs déjà construits ne l'avaient pas été avec le souci d'une optimisation de la pêche. Actuellement, avec l'essor des loisirs nautiques : pêche, voile, planche à voile... tout le monde est conscient qu'un aménagement hydraulique a beaucoup à gagner à prendre en compte ces activités. C'est ce qui s'est passé pour le barrage Aube.
Au point de vue piscicole, les principaux inconvénients rencontrés dans les réservoirs anciennement construits sont :

- le fond n'était que partiellement déboisé et pratiquement pas dessouché. La pêche de la carpe est impossible dans beaucoup d'endroits. La navigation et la pêche interdites de fait dans les parties non déboisées;

- des réservoirs à niveau constant ont été aménagés surtout pour les sports nautiques autres que la pêche. Les digues étaient à une cote inférieure à la cote maximale du plan d'eau. Le peuplement piscicole de ces plans est donc incontrôlable. Leur vidange est rendue difficile par un aménagement minimum de la pêcherie ;

- le profil du fond du réservoir n'a pas été aménagé de façon à avoir une vidange complète. Les emprises constituent des poches d'eau où le poisson se réfugie sans pouvoir être pêché. La vidange générale ne comporte pas de pêcherie fonctionnelle. On ne peut donc pas dans ces plans récupérer de façon correcte le poisson des vidanges décennales.

Compte tenu de ces faits : la pêche est orientée vers les poissons carnivores : brochet, perche, sandre. Le pêcheur de carpe ou le pêcheur de cyprinidés, en général, ne fréquente pas beaucoup ces plans d'eau en raison aussi des

(*) Président FDAAPP de l'Aube, Membre du comité de bassin Seine-Normandie, Président de la Commission de Bassin SeineNormandie, Membre du Comité de Pilotage pour l'Aménagement et la gestion du barrage Aube, Administrateur de l'Agence financière de bassin Seine-Normandie.

\section{A successful fish farming development, that of the Aube dam}

Over the last few decades, three main level control weirs were built upstream from Paris. The fish farming developments on large spreads of water came up against many difficulties. These did not escape the rule. The author analyses how the experience acquired on the first works has been able to be used to design and implement a coordinated fish farming development programme on the latest barrage built and how close collaboration between "developers" and "fishers" brought this about. A technical appendix brings together the descriptive and statistical elements. 
marnages fréquents qui déplacent le poisson et ne permettent pas "l'amorçage d'un coin de pêche ". Il y a alors un déséquilibre dans les peuplements piscicoles avec vieillissement des populations de Cyprinidés qui ne produisent plus suffisamment de jeunes poissons constituant le fourrage des prédateurs.

Le fait que les pêcheurs aient bien identifié les points importants à respecter pour rendre efficace un aménagement, a certainement permis un échange fructueux avec l'Institution des Barrages Réservoirs.

La présence permanente des pêcheurs aux nombreuses réunions d'élaboration du projet a donné une forte crédibilité à leur demande qui reposait sur une analyse résultant de l'expérience des réservoirs Marne et Seine.

Un programme de suivi permettra de lever les dernières incertitudes et d'ajuster, si nécessaire, la gestion piscicole de l'ouvrage.

\section{Les problèmes posés par la gestion piscicole des grands réservoirs}

\subsection{Un début euphorique des lendemains décevants}

A la mise en eau d'un réservoir, les terres inondées sont souvent riches et échangent avec l'eau des sels minéraux qui permettent une importante production, aussi bien végétale qu'animale, en fin de chaîne alimentaire. La pêche est alors très bonne. Quand les échanges entre l'eau et les sols se stabilisent, la production baisse et la satisfaction du pêcheur également. Le pêcheur est alors déçu, et par comparaison avec les premières années, affirme volontiers que le poisson n'existe plus. Il faut relativiser ceci et le faire comprendre au pêcheur.

\subsection{Les effets du marnage}

Le réservoir subit parfois des baisses de niveau importantes incompatibles avec le bon déroulement de certaines phases de la vie des poissons. Il s'agit en particulier de la reproduction de beaucoup d'espèces qui se déroule dans la zone littorale soumise au marnage.

Il faut quand même savoir que ce stade est assez souvent bref et bien synchronisé pour tous les individus d'une population. Une concertation pourrait être possible si les gestionnaires font le choix des espèces à aider et savent déterminer précisément le moment crucial où un marnage serait fatal.. Dans le cas des réservoirs Marne, Seine et Aube, la gestion de l'eau est en général compatible avec le bon déroulement des espèces, comme le brochet, très recherchées par le pêcheur.

\subsection{Un milieu souvent peu accessible à la pêche}

Le marnage, souvent important, ne facilite pas l'accès à la surface du plan d'eau d'autant plus que souvent le sol n'était pas aménagé. Quand des étendues de culture sont recouvertes, il y a très peu d'obstacles sur le fond. Mais quand il s'agit de forêts, le sol n'est pas souvent nettoyé. Quand des arbustes sont submergés, il n'y a aucune pêche possible. La pêche se limite à certaines surfaces, ce qui en soit n'est pas un mal, mais s'oriente seulement sur quelques espèces, délaissant des espèces de fond qui sont peu capturables dans ce cas. Les lignes s'accrochent trop facilement sur le fond et la pêche devient trop fastidieuse. Ces espèces vont ainsi proliférer.

\subsection{Des espèces indésirables difficiles à éliminer}

Dans les réservoirs Marne et Seine, se sont développées des espèces très peu recherchées par le pêcheur français. La Brème est certainement l'espèce qui pose le plus de problèmes. Les gros poissons âgés ne sont plus productifs et il est généralement admis qu'ils devraient être éliminés des plans d'eau. Hélas, même aux vidanges décennales, cette élimination est impossible. Les réservoirs ne sont pas totalement vidangeables. Les emprises, par exemple, ont été laissées telles quelles en fin d'exploitation et constituent des trous d'eau dans lesquels les poissons se réfugient et arrivent à survivre, en particulier la Brème.

\section{Les aménagements du réservoir Aube intègrent l'expérience acquise sur les réservoirs anciens}

\subsection{L'aménagement complet du fond}

\section{a) Le profil}

Au réservoir Aube, toutes les emprises ont été réalisées de façon à ne pas créer de poches d'eau non vidangeables. De plus, des drains ont été réalisés pour faciliter le rassemblement du poisson dans le fond de la retenue avec création d'une surface d'eau résiduelle en amont des ouvrages des digues.

Lors d'une vidange, le poisson peut ainsi se rassembler dans une pièce d'eau qu'il sera possible ultérieurement de vidanger si on veut effectivement intervenir sur le peuplement piscicole en éliminant une espèce indésirable (Brème, poisson-chat...). Il y a ainsi dissociation entre la vidange pour la surveillance des ouvrages et une vidange complète pour accéder à tout le peuplement piscicole.

\section{b) Dessouchage}

Le réservoir Aube est implanté essentiellement sur une forêt. Non seulement le sol a été complètement nettoyé mais les souches ont été arrachées. Les débris de bois ont été mis en andains et brûlés. Les résidus ont été enterrés dans des tranchées profondes et la surface du sol aplanie. Cet aménagement permet d'accéder à toute la surface du fond pour la pêche mais n'a pas que ce bénéfice. Sur un plan esthétique, il est de beaucoup préférable d'avoir un fond propre qu'un fond d'où sortent des souches quand le plan d'eau est à un niveau bas. L'aménagement piscicole rejoint dans ce cas des aménagements touristiques. 


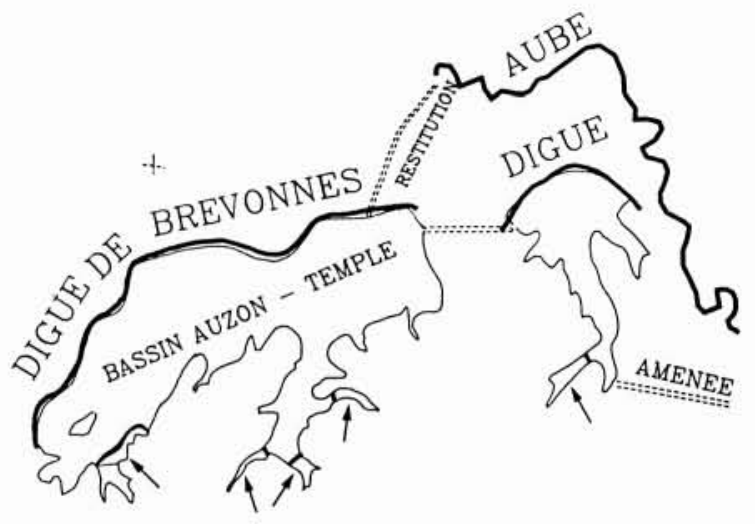

1. Les aménagements du réservoir Aube.

$\rightarrow$ Plans d'eau à niveau constant prévus pour la gestion piscicole.

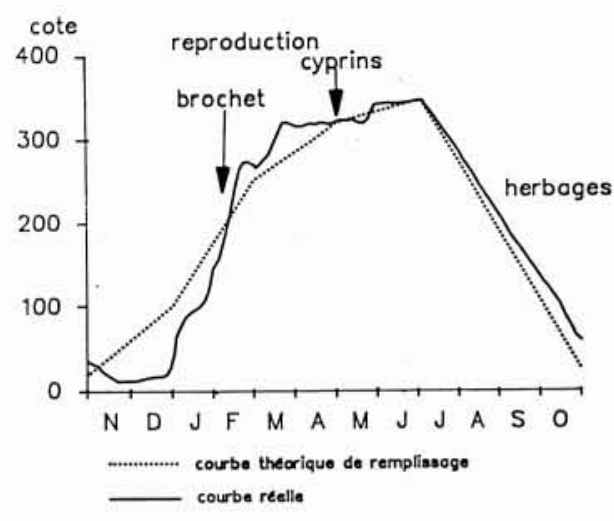

2. Gestion de l'eau d'un réservoir Seine, Marne ou Aube. L'évolution du marnage est compatible avec la vie piscicole.

\subsection{Des retenues à niveau constant}

Le contour du réservoir plein est très digité et les « queues de retenues " sont nombreuses. Dans le cas du barrage Marne, par exemple deux plans d'eau à niveau constant avaient été aménagés surtout dans un but de loisirs nautiques autre que la pêche. En effet, les digues de ces plans d'eau sont inondables aux plus hauts niveaux d'eau. On ne peut donc pas contrôler le peuplement piscicole. Un empoissonnement particulier risque d'être inefficace si peu de temps après la retenue est en relation avec l'ensemble du réservoir. Dans le cas du réservoir Aube, le contexte est beaucoup plus propice. Le plan d'eau Amance sera maintenu avec un faible marnage et destiné aux sports nautiques. Les queues de retenues ont ainsi pu être aménagées à la seule fin halieutique et donc les digues ont été construites de façon à n'être jamais submergées. On peut donc réaliser une gestion piscicole séparée de ces différents plans d'eau.

\subsection{Des pêcheries sur tous les ouvrages}

Tous les ouvrages ont été équipés de pêcherie pour recueillir dans de bonnes conditions les poissons lors des vidanges. Cet aménagement n'est concevable que si des unités de stockage existent parallèlement. La fédération a acquis à cet effet des plans d'eau à proximité (complexe piscicole de Joncheric : 21 hectares d'étangs et de bassins de stockage).

\subsection{Un coût financier non négligeable}

Le coût total de ces aménagements s'élève à $17 \mathrm{MF}$. Il faut toutefois relativiser ceci. En pourcentage du coût global de la construction du réservoir, les aménagements piscicoles représentent $1 \%$. Ces aménagements seraient beaucoup plus coûteux s'ils devaient être faits après la construction du réservoir et l'expérience montre qu'ils ne sont souvent plus réalisés. Une bonne concertation a permis dans ce cas de prendre en compte tous les aspects de la construction du réservoir.

\section{Conclusion}

La construction du réservoir Aube est un exemple de prise en compte, dès la réalisation, de tous les aspects socioéconomiques liés à un tel aménagement. L'aspect piscicole a ainsi trouvé sa place au même titre que les autres aspects comme les sports nautiques. Cet aménagement devrait avoir des conséquences sur le plan de l'ouverture touristique de cette région qui va être, grâce aux nouvelles autoroutes, très proche de Paris aussi bien que de la Belgique.

Ces aménagements réellement efficaces ont motivé des travaux de recherche et développement concernant le plan de gestion piscicole qui reposera sur un programme de suivi. Des statistiques de pêche seront mises en place pour connaître le niveau de la pêche. Parallèlement des méthodes modernes comme l'échosondage apporteront une bonne connaissance du peuplement en place. 


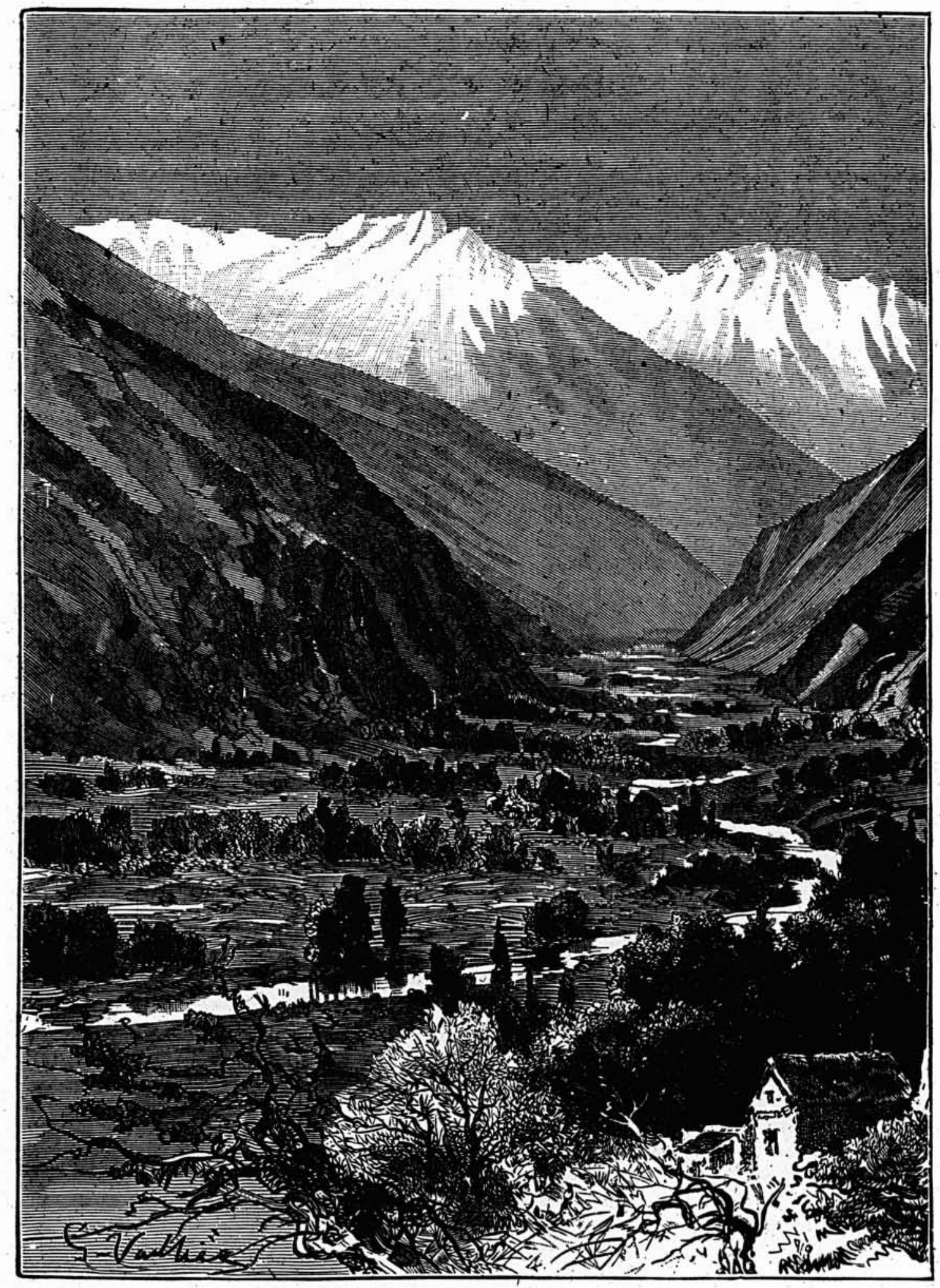

